\title{
Comparing efficacy, safety and stability of Femtosecond assisted LASIK and implantable collamer lens implantation in correction of high myopia
}

\author{
ElshimaaA.Mateen, Ahmad Mustafa Abd Allah, HatemGamalAmmar, Engy \\ Mohammad Mustafa. \\ Ophthalmology department, Faculty of medicine, Sohag University.
}

\begin{abstract}
PURPOSE: To compare efficacy, safety and stability of Femtosecond assisted LASIK and implantable collamer lens implantation in correction of high myopia.

METHODS: This study was non-randomized comparative prospective consecutive interventional study. It included 74 eyes with high myopia ( $\geq-6$ Ds) of 40 patients attended to the outpatient ophthalmic clinic of Sohag university hospitals from the period from Jan.2016 to Jan.2017.

The patients were divided into two groups depending on: Degree of myopia, corneal thickness and corneal tomography.

The first group (34 eyes) of 20 patients were subjected to implantable collamer lenses implantation and the second group (40 eyes) of 40 patients were subjected to femtosecond assisted LASIK.

RESULTS: Group one (ICL group) showed UCVA preoperative was (1.90 \pm 0.29$)$ and UCVA postoperative was $(0.27 \pm 0.21)$ with (p-value $\left.<0.000^{*}\right)$. BCVA preoperative was $(0.526 \pm 0.272)$ and that of BCVA postoperative was $(0.217 \pm 0.128)$ with (p-value $<0.001 *)$, which means that BCVA postoperative was better than what was expected from the BCVA preoperative.

Spherical error decreased from $(-13.576 \pm 3.945)$ preoperative to $(-0.0385 \pm 0.821)$ postoperative with $(\mathrm{p}$-value $<0.000 *)$. Cylindrical error preoperative was $(-1.134 \pm 0.617)$ and postoperative was $(-0.352 \pm 0.250)$ with $(\mathrm{p}$-value $<0.000 *)$.Spherical equivalent preoperative was $(-15.173 \pm 4.079)$ and that of postoperative was $(-0.269 \pm 0.787)$ with (p-value $\left.<0.000^{*}\right)$.

Group two (Femtosecond assisted LASIK) UCVA preoperative was (1.42 \pm 0.27$)$ and UCVA postoperative was $(0.366 \pm 0.21)$ with $\left(\mathrm{p}\right.$-value $\left.<0.000^{*}\right)$. There wasn't significant difference between BCVA preoperative and postoperative. Spherical error preoperative was ($8.398 \pm 1.733)$ and postoperative $(-0.796 \pm 1.186)$ with (p-value $<0.000 *)$. Cylinder error mean value preoperative was $(-1.648 \pm 0.838)$ corrected to $(-0.765 \pm 0.423)$ with (p-value< $0.005)$.Spherical equivalent preoperative was $(-9.213 \pm 1.899)$ and that of postoperative is ($0.935 \pm 1.11$ ) with (p-value $<0.000 *$ ).

CONCLUSIONS: Both implantable collamer lens and Femtosecond assisted LASIK proved high efficacy and safety but implantable collamer lens showed more stability for high myopic patients.
\end{abstract}

Keywords: High myopia, Femtosecond, Excimer laser, Implantable collamer lens , efficacy, safety, stability.

\section{Introduction:}

Myopia often known as "being short sighted" causes vision to be blurry in the distance but clearer when looking at things up close. Myopia eye which bends the coming light too much, which means that the light comes to a focus point before it reaches the retina. When moving closer to an object, this changes the focusing of the light and the object is then in focus on the retina and therefore looks clear.

Myopia depends on:

- The length of the eyeball from front to back

- How steep the cornea is

- How powerful the lens is.

Classification of myopia (1):

- Mild myopia includes powers up to 3.00 diopters (D) 
- Moderate myopia, values of -3.00 to $-6.00 \mathrm{D}$

- High myopia is usually myopia over $-6.00 \mathrm{D}$

Phakic intraocular lenses (PIOLs) are generally accepted as an alternative treatment for ametropia correction among various refractive ranges. Fast visual recovery, high efficacy, predictability and stability of visual quality, preservation of accommodation, and reversibility are several advantages that have been attributed to PIOL implantation. $(2,3)$

The Visian Implantable Collamer Lens (ICL; STAAR Surgical Co, Monrovia, California) is approved by the United States Food and Drug Administration (FDA) for the treatment of moderate to severe myopia. The lens material, trade-named Collamer, is a hydrophilic collagen-polymer combination with a water content of $34 \%$ and a refractive index of 1.45. (2)

ICL implantation can correct myopia $(3,4)$, hyperopia $(5,6)$, or astigmatism(7, 8), with clinical and visual results as good as or better than laser procedures $(7,8)$. Patients who are not suitable candidates for corneal reshaping procedures, and in whom optical correction with spectacles or contact lenses is either challenging or have poor results, $(9,10)$ can benefit from ICL surgery.

Although the ICL offers outstanding advantages, there have been reports in the literature of postoperative complications associated with both high and low degrees of vault of the ICL over the crystalline lens. Low vault may lead to mechanical contact with the crystalline lens or inadequate aqueous circulation, which is responsible for a high incidence of anterior capsular opacification and cataract formation (11, 12). Conversely, excessively high vault causes mechanical contact between the ICL and the iris, resulting in inflammation, high intraocular pressure, angle-closure glaucoma and pigment dispersion syndrome (13). Recently, a new implantable collamer lens (ICL V4c), with a $360 \mu \mathrm{m}$ central hole that allows for the natural flow of aqueous humor without the need for a peripheral iridotomy, which may reduce the risk of anterior capsular opacification and cataract formation comparing to the old forms of ICL(14, 15) . Previous studies have shown that the pupil constriction in response to light can affect the vault, eventually causing the ICL to move posteriorly towards the crystalline lens, leading to a significant decrease in central vault under photopic conditions $(9,14)$. Du et al. reported that the distance between the ICL and the crystalline lens reduced as the ICL was moved posteriorly by the iris as a result of pupil constriction during pharmacologic accommodation with topical pilocarpine. Simultaneously, the anterior surface of the crystalline lens became more convex and moved anteriorly, further reducing the central vault of the ICL (15). Furthermore, it has been reported that vault has a tendency to decrease over time, along with physiologic increase of lens thickness with age. $(3,13,16)$

Femtosecond (FS) laser is an infrared laser with a wavelength of $1053 \mathrm{~nm}$. It works by producing photodisruption or photoionization of the optically transparent tissue such as the cornea.(1) Application of FS results in the generation of a rapidly expanding cloud of free electrons and ionized molecules. The acoustic shock wave generated results in disruption of the treated tissue. (17)

FS laser has pulse duration in the femtosecond range (10-15 second). Reducing the pulse duration reduces the amount of collateral tissue damage which makes FS laser safe to be used in corneal surgeries. $(18,19)$ 
Flap creation using (FS), the suction ring is centered over the pupil and suction is applied once the proper centration of the ring has been ensured . The docking procedure is then initiated while keeping the suction ring parallel to the eye. An applanating glass contact lens is used to stabilize the globe and to flatten the cornea. It is important to achieve complete applanation of the cornea to avoid an incomplete flap or other flap related complications. Once the laser's computer has confirmed centration, the surgeon administers the FS laser treatment. Each pulse of the laser generates free electrons and ionized molecules leading to formation of microscopic gas bubbles dissipating into surrounding tissue. Multiple pulses are applied next to each other to create a cleavage plane and ultimately the LASIK flap. Suction is then released. A spatula is carefully passed across the flap starting at the hinge and sweeping inferiorly to lift the flap for excimer laser ablation.(17, 20-22)

\section{METHODS}

Subjects

Fourty individuals (34 eyes) subjected to implantable collamer lens implantation surgery and (40 eyes) subjected to femtosecond assisted Lasik surgery, aged 27-33 years who attended to the outpatient ophthalmic clinic of Sohag university hospitals from the period from Jan.2016 to Jan.2017.Group one (ICL) had Spherical refractive errors mean value $-13.576 \mathrm{D}( \pm 3.945)$ with astigmatism $1.134 \mathrm{D}( \pm 0.617)$. The patients had clear intraocular media and no known ocular pathology. Group two (Femto second assisted Lasik) had Spherical refractive errors mean value $8.398( \pm 1.733)$ with astigmatism $1.648( \pm 0.838)$. The patients had clear intraocular media and no known ocular pathology.
The tenets of the declaration of Helsinki were followed. Informed consent was obtained from each participant after verbal and written explanations of the nature and possible consequences of the study were provided .The study protocol received institutional review board approval.

\section{INTRAOCULAR LENS}

The Visian ICL is a plate-haptic single-piece intraocular lens, which is a flexible. It can be folded and implanted in the posterior chamber via a $2.8-3.2 \mathrm{~mm}$ corneal incision. It has a high degree of biocompatibility, good permeability of gases and metabolites, and good absorption of ultraviolet radiation. The ICL design has been modified many times in the past. In this study, the phakic IOL patients were ICL V4c lens designs. The ICL $\mathrm{V} 4 \mathrm{c}$ is a $6.00 \mathrm{~mm}$ wide lens and comes in four sizes (12.1, 12.6, 13.2 and 13.7 $\mathrm{mm}$ in length). Its optic zone diameter is $4.9-5.8 \mathrm{~mm}$, with a spherical power range of -0.50 to $-18.00 \mathrm{DS}$ and a cylindrical power range of +0.50 to +6.00 DC. ICL power calculations were performed by the manufacturer (STAAR Surgical) using a modified vertex formula. The variables in the formula included preoperative manifest spherical and cycloplegic refractions, keratometric power, central corneal thickness and central ACD (ACD, Pentacam, measured from the corneal endothelium to the anterior lens). The size (length) of the implanted ICL was determined based on the patient's WTW and ACD. For the ICL V4c, the sizes (lengths) of 12.1, 12.6, 13.2 and $13.7 \mathrm{~mm}$ were equal to the ICL V4 sizes (lengths) of 11.5, 12.0, 12.5 and $13.0 \mathrm{~mm}$, respectively. (23)

\section{Femtosecond LASIK in high myopia:}

One of the concerns in the application of LASIK for highly myopic patients is the risk of post-LASIK ectasia 41,42 which is believed to be reduced 
SOHAG MEDICAL JOURNAL Vol. 22 No.3 October 2018 using femtosecond laser (femtoLASIK) and the ability of creation of smaller flap thickness. Less higher order aberrations (HOAs( are induced with femto-LASIK compared to the conventional approach.(24)Although concerns are not resolved completely, especially in cases with larger pupil diameters,(25) results in terms of contrast sensitivity, especially in high spatial frequencies, are better with femto-LASIK compared to the conventional method. $(26,27)$

Efficacy: Percentage of eyes with uncorrected visual acuity (UCVA) of 20/20 and 20/40 (2) Efficacy index, which is the ratio of the mean postoperative UCVA to the mean preoperative BCVA (i.e. mean postop. UCVA / mean preop.BCVA). (This is most easily calculated by converting the values of geometric mean acuities to decimal values)This measure is particularly useful in describing outcomes of high myopia when the

\section{RESULTS}

preoperative BCVA is worse than 20/20. (28)

Safety: Number and percentage of eyes losing two or more lines of best spectacle corrected visual acuity BCVA. (2) Safety index, which is the ratio of mean $\mathrm{BCVA}$ over mean preop. BCVA (i.e. Mean postop.BCVA / mean preop. BCVA) this is most easily calculated by converting the values of geometric mean acuities to decimal values.(28)

Stability: The number and percentage of eyes with a change of spherical equivalent of manifest refraction $\geq$ $1.00 \mathrm{D}$ within a specified interval, the recommended minimal interval is 6 months(28).

\section{DATA ANALYSIS}

Statistical analysis was performed using SPSS version 16(IBM, USA). An independent samples t-test was used to compare mean values of measured parameters. Pearson's correlation coefficient was used to evaluate the correlation between quantitative variables

Group one (ICL) showed UCVA preoperative was (1.90 \pm 0.29$)$ and UCVA postoperative was $(0.27 \pm 0.21)$ with (p-value $<0.000 *)$. BCVA preoperative was $(0.526 \pm 0.272)$ and that of BCVA postoperative was $(0.217 \pm 0.128)$ with (pvalue $<0.001 *)$, which means that BCVA postoperative was better than what was expected from the BCVA preoperative.

Spherical error decreased from $(-13.576 \pm 3.945)$ preoperative to $(-0.0385 \pm 0.821)$ postoperative with (p-value $<0.000 *)$.Cylindrical error preoperative was ($1.134 \pm 0.617)$ and postoperative was $(-0.352 \pm 0.250)$ with (p-value $<0.000 *)$.Spherical equivalent preoperative was $(-15.173 \pm 4.079)$ and that of postoperative was ($0.269 \pm 0.787$ ) with (p-value $\left.<0.000^{*}\right)$.

Group two (Femtosecond assisted Lasik) showed UCVA preoperative was $(1.42 \pm 0.27)$ and UCVA postoperative was $(0.366 \pm 0.21)$ with $(\mathrm{p}$-value $<0.000 *)$. There wasn't significant difference between BCVA preoperative and postoperative. Spherical error preoperative was $(-8.398 \pm 1.733)$ and postoperative $(-0.796 \pm 1.186)$ with $\left(\mathrm{p}\right.$-value $\left.<0.000^{*}\right)$. Cylinder error mean value preoperative was $(-1.648 \pm 0.838)$ corrected to $(-0.765 \pm 0.423)$ with ( $\mathrm{p}$-value $<0.005)$. Spherical equivalent preoperative was $(-9.213 \pm 1.899)$ and that of postoperative is $(-0.935 \pm 1.11)$ with ( $\mathrm{p}$-value $<0.000 *)$. There wasn't significant difference in UCVA, BCVA, spherical error, cylindrical error or spherical equivalent preoperatively between both groups.

There wasn't significant difference in UCVA and BCVA in both groups. Spherical error in group1 $(0.321 \pm 0.590)$ and group2 $(-0.75 \pm 0.31)$ with (p-value $<0.001 *)$ .Cylindrical error in group1 $(-0.392 \pm 0.318)$ and in group2(-0.750 \pm 0.204$)$ with (p- 
SOHAG MEDICAL JOURNAL Vol. 22 No.3 October 2018

value $<0.00 *)$. Spherical equivalent mean value of group1 $(0.0000 \pm 0.433)$, in group2 $(-1.392 \pm 0.748)$ (p-value $<0.001 *)$.

\section{Postoperative efficacy and safety of both groups:}

\begin{tabular}{|l|l|l|}
\hline & $\begin{array}{l}\text { Group one(ICL) } \\
(\mathrm{n}=34)\end{array}$ & $\begin{array}{l}\text { Group two(Femtosecond Lasik) } \\
(\mathrm{n}=40)\end{array}$ \\
\hline Efficacy & 0.95 & 1.01 \\
\hline Safety & 1.9 & 0.98 \\
\hline
\end{tabular}

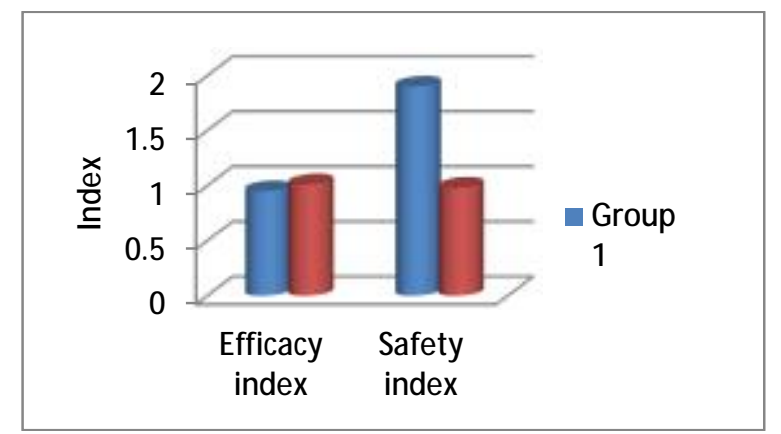

Both groups showed high efficacy, but Group1 showed more safety.

Group1 showed $100 \%$ stability while group2 showed $71.4 \%$, as shown in the below graph.

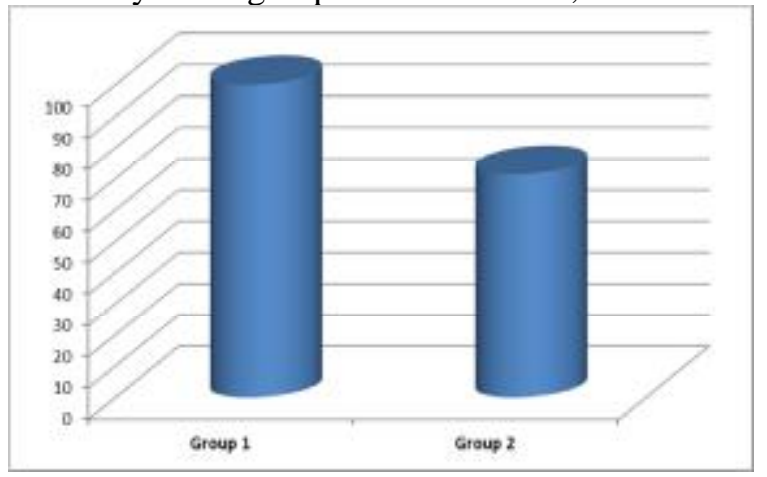

Graph showing difference in stability between both groups

\section{DISCUSSION}

The aim of this study was to evaluate the visual outcomes of femtosecond LASIK in comparison with implantable collamer lenses for correction of high myopia regarding visual acuity, high order aberrations, contrast sensitivity, efficacy, safety and stability.

The patients were divided into two groups .The first group was subjected to implantable collamer lenses implantation and the second group subjected to femtosecond assisted LASIK.

Excimer laser surgeries proved effectiveness for myopia correction, but they have complications such as myopic regression and ectasia which were reported in eyes with high myopia(29, 30).Correction of high myopia is associated with increased HOAs(31).

It is proved that Phakic IOL implantation has predictability in correction of high myopia (32, 33).Implantation of PIOL can induce complications such as cataract, lens dislocation and elevation of intraocular pressure. Therefore ,assessing visual and outcomes of PIOL is helpful when selecting the more appropriate, safe ,stable and effective procedure to 
correct high myopia, especially when the patients have an overlapping range of both procedures(34).

Our study showed excellent refractive and visual outcomes with both ICL implantation and Femtosecond assisted LASIK.

The study showed that with a pupillary diameter 3-mm, VA values are improved in $100 \%$ of cases which can be up to $(0.1 \log$ MAR or more $)$ after 6 months follow up $(\mathrm{p}<0.000 *)$. There is agreement between our results and results of Paul and Taylor who studied refractive outcomes and safety of the implantable collamer lens in young low to moderate myopes. Their study was retrospective study performed by chart review of (104 eyes) with 50 months follow up period (35).

The results of group two (Femtosecond assisted LASIK group)from the visual quality point of view with a pupillary diameter 4-mm, VA values improved in $100 \%$ of cases which is up to $(0.1$ $\log$ MAR or more ) after 6 months follow up, evaluated $\left(\mathrm{p}<0.00^{*}\right)$. This result is consistent with that of Cari Perez-Vives et al(36).Contrast sensitivity in photopic conditions increased comparing preoperative BCVA and postoperative BCVA with $\left(p<0.007^{*}\right)$. This result is consistent with Cari Perez -Vives et al. and JOO youn SHIN, et al $(34,36)$.

Both groups showed high efficacy and safety, but group one (ICL) showed more stability during the follow up period than group two (Femtosecond Lasik). These finding were consistent with that of Xun Chen et, al. who studied contralateral eye comparison of the long-term visual quality and stability between implantable collamer lens and laser refractive surgery for myopia. Their study conducted on 52 eyes of 26 high-myopia anisometropia patients who were suitable for surgical treatment. In each patient, the higher-myopia eye was implanted with
ICL and the lower-myopia eye was treated with LRS. The patients were followed for 3 years(37).

As regards complications in this study:

Complications can be divided into intraoperative and postoperative complications.

\section{1)Intraoperative complications:}

\section{Group 1}

-One eye ICL implanted upside down with partially torn haptic during manipulations for removal and reinsertion. It is a rare complication of ICL implantation which is mentioned by Amar Agarwal and Kumar, who studied Visco-cannula assists in reinversion of phakic lens (38)

\section{Group2}

-Three eyes showed decentered ablations detected during follow up. This complication is stated by Hiroko Biessen-Miyajima , a book of cataract and refractive surgery (39).

\section{2) Postoperative complications: Group1}

-One eye developed cataract. This complications is also mentioned by Seyed Javad Hashemian,MD,et al studied the outcomes and complications of ICL and toric ICL for the correction of high myopia with and without astigmatism ,whose study involved 95 eyes and followed up for one year (40) .

-One eye developed postoperative iritis which can be attributed to the manipulations made to remove and reinsert the lens intraoperative

-Three eyes were steroid responders and IOP improved by cessation of steroids. This complication is encountered by SirishSenthil, et al. who studied etiology and management of raised intraocular pressure following posterior chamber phakic intraocular lens implantation in myopic eyes. They studied 638 eyes between 2009 and 2015(41) .

\section{Group2}


-One eye showed DLK which was improved by steroids. This complication was encountered by Ayad A.Farjo ,MD,et al. who studied femtosecond lasers for LASIK flap creation, a report by the American academy of ophthalmology (42).

In summary both Implantable collamer lens implantation and femtosecond assisted Lasik for high myopia proved high efficacy and safety but ICL showed more stability during the follow up period.

\section{References:}

1. Chung SH, Mazur E. Surgical applications of femtosecond lasers. Journal of biophotonics. 2009;2(10):557-72.

2. Lovisolo CF, Reinstein DZ. Phakic intraocular lenses. Survey of ophthalmology. 2005;50(6):549-87.

3. Sanders DR, Doney K, Poco M. United States Food and Drug Administration clinical trial of the Implantable Collamer Lens (ICL) for moderate to high myopia: three-year follow-up. Ophthalmology. 2004;111(9):1683-92.

4. Lackner B, Pieh S, Schmidinger G, Simader C, Franz C, DejacoRuhswurm I, et al. Long-term results of implantation of phakic posterior chamber intraocular lenses. Journal of cataract and refractive surgery. 2004;30(11):2269-76.

5. Bloomenstein MR, Dulaney DD, Barnet RW, Perkins SA. Posterior chamber phakic intraocular lens for moderate myopia and hyperopia. Optometry ( $\mathrm{St}$ Louis, Mo). 2002;73(7):435-46.

6. Pesando PM, Ghiringhello MP, Di Meglio G, Fanton G. Posterior chamber phakic intraocular lens (ICL) for hyperopia: Ten-year follow-up. Journal of Cataract \& Refractive Surgery. 2007;33(9):1579-84.

7. Sanders D, Vukich JA. Comparison of implantable collamer lens (ICL) and laser-assisted in situ keratomileusis (LASIK) for low myopia. Cornea. 2006;25(10):1139-46.

8. Schallhorn S, Tanzer D, Sanders DR, Sanders ML. Randomized prospective comparison of visian toric implantable collamer lens and conventional photorefractive keratectomy for moderate to high myopic astigmatism. Journal of refractive surgery (Thorofare, NJ : 1995). 2007;23(9):853-67.

9. Kamiya K, Shimizu K, Hikita F, Komatsu M. Posterior chamber toric phakic intraocular lens implantation for high myopic astigmatism in eyes with pellucid marginal degeneration. Journal of cataract and refractive surgery. 2010;36(1):164-6.

10. Lesueur LC, Arne JL. Phakic posterior chamber lens implantation in children with high myopia. Journal of cataract and refractive surgery. 1999;25(12):1571-5.

11. Sanders DR, Vukich JA, Doney K, Gaston M. U.S. Food and Drug Administration clinical trial of the Implantable Contact Lens for moderate to high myopia. Ophthalmology. 2003;110(2):255-66.

12. Schmidinger G, Lackner B, Pieh $S$, Skorpik C. Long-term changes in posterior chamber phakic intraocular collamer lens vaulting in myopic patients. Ophthalmology. 2010;117(8):1506-11.

13. Lackner B, Pieh S, Schmidinger G, Hanselmayer G, Dejaco-Ruhswurm I, Funovics MA, et al. Outcome after treatment of ametropia with implantable contact lenses. Ophthalmology. 2003;110(11):215361.

14. Lee H, Kang SY, Seo KY, Chung B, Choi JY, Kim KS, et al. Dynamic vaulting changes in $\mathrm{V} 4 \mathrm{c}$ versus $\mathrm{V} 4$ posterior chamber phakic lenses under differing lighting conditions. Am J Ophthalmol. 2014;158(6):1199-204.e1.

15. Du C, Wang J, Wang X, Dong $Y, G u$ Y, Shen Y. Ultrasound biomicroscopy of anterior segment accommodative changes with posterior chamber phakic intraocular lens in high myopia. Ophthalmology. 2012;119(1):99-105.

16. Baumeister M, Buhren J, Kohnen T. Position of angle-supported, irisfixated, and ciliary sulcus-implanted myopic phakic intraocular lenses evaluated by Scheimpflug 
photography. Am J Ophthalmol. 2004;138(5):723-31.

17. Soong HK, Malta JB. Femtosecond lasers in ophthalmology. Am J Ophthalmol. 2009;147(2):189-97.e2.

18. Stern D, Schoenlein RW, Puliafito CA, Dobi ET, Birngruber R, Fujimoto JG. Corneal ablation by nanosecond, picosecond, and femtosecond lasers at 532 and $625 \mathrm{~nm}$. Archives of ophthalmology (Chicago, Ill : 1960). 1989;107(4):587-92.

19. Ratkay-Traub I, Ferincz IE, Juhasz T, Kurtz RM, Krueger RR. First clinical results with the femtosecond neodynium-glass laser in refractive surgery. Journal of refractive surgery (Thorofare, NJ : 1995). 2003;19(2):94103.

20.Durrie DS, Kezirian GM. Femtosecond laser versus mechanical keratome flaps in wavefront-guided laser in situ keratomileusis: prospective contralateral eye study. Journal of cataract and refractive surgery. 2005;31(1):120-6.

21. Slade SG. The use of the femtosecond laser in the customization of corneal flaps in laser in situ keratomileusis. Current opinion in ophthalmology. 2007;18(4):314-7.

22.Kymionis GD, Kankariya VP, Plaka AD, Reinstein DZ. Femtosecond laser technology in corneal refractive surgery: a review. Journal of refractive surgery (Thorofare, NJ : 1995). 2012;28(12):912-20.

23. Chen $X$, Miao H, Naidu RK, Wang X, Zhou X. Comparison of early changes in and factors affecting vault following posterior chamber phakic Implantable Collamer Lens implantation without and with a central hole (ICL V4 and ICL V4c). BMC Ophthalmology. 2016;16(1):161.

24. Samuel A-M, Diego DO. Analysis of optimized profiles for 'aberration-free' refractive surgery. Ophthalmic and Physiological Optics. 2009;29(5):53548.

25.Vega-Estrada A, Alio JL, Arba Mosquera S, Moreno LJ. Corneal higher order aberrations after LASIK for high myopia with a fast repetition rate excimer laser, optimized ablation profile, and femtosecond laser-assisted flap. Journal of refractive surgery (Thorofare, NJ : 1995). 2012;28(10):689-96.

26.Lim $T$, Yang $S$, Kim $M$, Tchah $H$. Comparison of the IntraLase femtosecond laser and mechanical microkeratome for laser in situ keratomileusis. Am J Ophthalmol. 2006;141(5):833-9.

27.Montés-Micó R, Rodríguez-Galietero A, Alió JL, Cerviño A. Contrast sensitivity after LASIK flap creation with a femtosecond laser and a mechanical microkeratome. Journal of refractive surgery (Thorofare, NJ : 1995). 2007;23(2):188-92.

28. Koch DD, Kohnen T, Obstbaum SA, Rosen ES. Format for reporting refractive surgical data. Journal of cataract and refractive surgery. 1998;24(3):285-7.

29. Krueger RR, Talamo JH, McDonald $\mathrm{MB}$, Varnell RJ, Wagoner MD, McDonnell PJ. Clinical Analysis of Excimer Laser Photorefractive Keratectomy Using a Multiple Zone Technique for Severe Myopia. American Journal of Ophthalmology. 1995;119(3):263-74.

30. Stulting RD, Carr JD, Thompson KP, Waring GO, Wiley WM, Walker JG. Complications of laser in situ keratomileusis for the correction of myopia. Ophthalmology. 1999;106(1):13-20.

31. Yamane N, Miyata K, Samejima T, Hiraoka T, Kiuchi T, Okamoto F, et al. Ocular Higher-Order Aberrations and Contrast Sensitivity after Conventional Laser In Situ Keratomileusis. Investigative Ophthalmology \& Visual Science. 2004;45(11):3986-90.

32. Igarashi A, Kamiya K, Shimizu K, Komatsu M. Visual Performance after Implantable Collamer Lens Implantation and Wavefront-Guided Laser In Situ Keratomileusis for High Myopia. American Journal of Ophthalmology. 2009;148(1):16470.e1.

33. Sanders DR, Vukich JA. Comparison of implantable contact lens and laser 
assisted in situ keratomileusis for moderate to high myopia. Cornea. 2003;22(4):324-31.

34. Shin JY, Ahn H, Seo KY, Kim EK, Kim TI. Comparison of higher order aberrations after implantable Collamer Lens implantation and wavefrontguided LASEK in high myopia. Journal of refractive surgery (Thorofare, NJ : 1995). 2012;28(2):106-11.

35.Dougherty PJ, Priver T. Refractive outcomes and safety of the implantable collamer lens in young low-tomoderate myopes. Clin Ophthalmol. 2017;11:273-7.

36. Perez-Vives $\mathrm{C}$, Albarran-Diego $\mathrm{C}$, Garcia-Lazaro S, Ferrer-Blasco T, Montes-Mico R. Implantable collamer lens and femtosecond laser for myopia: comparison using an adaptive optics visual simulator. Arquivos brasileiros de oftalmologia. 2014;77(2):103-9.

37.Chen X, Guo L, Han T, Wu L, Wang $\mathrm{X}$, Zhou $\mathrm{X}$. Contralateral eye comparison of the long-term visual quality and stability between implantable collamer lens and laser refractive surgery for myopia. Acta ophthalmologica. 2018.
38. Amar Agarwal M, Kumar DA. Viscocannula assists in reinversion of phakic lens. Ocular Surgery News. 2009;20(4):25.

39. Bissen-Miyajima H. Complications of Excimer Laser Surgery. Cataract and Refractive Surgery: Springer; 2006. p. 101-11.

40.Hashemian SJ, Bigzadeh F, Foroutan A, Tajoddini S, Ghaempanah MJ, Jafari ME. Outcomes and Complications of Implantable Collamer Lens and Toric Implantable Collamer Lens for the Correction of High Myopia with and without Astigmatism (One Year Prospective Study). Iranian Journal of Ophthalmology. 2013;25(1):8.

41. Senthil S, Choudhari NS, Vaddavalli PK, Murthy S, Reddy J, Garudadri CS. Etiology and management of raised intraocular pressure following posterior chamber phakic intraocular lens implantation in myopic eyes. PloS one. 2016;11(11):e0165469.

42.Farjo AA, Sugar A, Schallhorn SC, Majmudar PA, Tanzer DJ, Trattler WB, et al. Femtosecond lasers for LASIK flap creation: a report by the American Academy of Ophthalmology. Ophthalmology. 2013;120(3):e5-e20. 
SOHAG MEDICAL JOURNAL Comparing efficacy, safety and stability of Femtosecond assisted 